Research Article

\title{
Two Improved Cancellation Techniques for Direct-Conversion Receivers
}

\author{
Xueyuan Hao and Xiaohong Yan \\ Nanjing University of Posts and Telecommunications, Nanjing 210003, China \\ Correspondence should be addressed to Xueyuan Hao; haoxy@njupt.edu.cn
}

Received 19 April 2016; Revised 15 July 2016; Accepted 27 July 2016

Academic Editor: Ahmed M. Soliman

Copyright (c) 2016 X. Hao and X. Yan. This is an open access article distributed under the Creative Commons Attribution License, which permits unrestricted use, distribution, and reproduction in any medium, provided the original work is properly cited.

To solve the problems of carrier leakage and DC offset in direct-conversion receiver (DCR) system, the paper proposed two kinds of improved technology to overcome the problems in DCR system. One is the RF carrier cancellation technology; the traditional cancellation technology based on lumped parameter filter can be easily influenced by distribution parameters, the improved circuits use a $3 \mathrm{db}$ bridge to realize a 180-degree phase shifter, and the method can adapt to a wider range of RF frequency. Another is DC offset cancellation technique; a novel DC servo loop circuit is proposed to replace the traditional AC-coupled amplifier circuit. It can improve the integrity of the baseband signal and reduces the complexity of the subsequent software algorithm. Experimental results show that two kinds of improved technology can improve the performance of DCR and expand its scope of application.

\section{Introduction}

The direct-conversion receiver (DCR), also known as zero-IF receiver, is a radio receiver design that realizes the RF signal one-time conversion to baseband signal. Compared to the super heterodyne structure, the DCR system has no mirror frequency interference and can be easily realized with low cost [1].

In the single antenna DCR system, a special component, the circulator, is generally used to realize the isolation between sending signals and receiving signal; it is shown in Figure 1.

Circulator is a three-port device, including the launch port, the antenna port, and a receive port. Due to the properties of the magnetic material, the circulator is not easy to be designed with high isolation. Measured by the vector network analyzer in good match case, the isolation can only reach $26 \mathrm{~dB}$. In actual application, due to wiring length in circuit board and the antenna impedance mismatch, the isolation is often lower than $20 \mathrm{~dB}$ [2]. The low isolation will cause some problems, the first one is linearity issue; the transmitted signal can be leaked into the receive port by several ways as shown in Figure 2; one way is through the antenna port directly leaking into the receive port, and the other one is from the launch port circulator leakage to the receiving port. The power of leakage signal in receiving port is far greater than the received signal power; it is easy to cause the receiver front-end circuit (LNA and mixer) saturation.

Another issue of the DCR system is the DC offset problem. Any leakage between LO and RF ports of the mixer will produce an undesired DC component; the large DC signal will stature the subsequent DC amplifier. Because of the existence of the DC voltage, the wideband amplifier cannot work in DC coupling mode and can only work in the AC coupling mode. The AC capacitor not only limits the data rate but also influences the pulse width of the baseband signal, and the uncertain baseband signal pulse width will seriously affect the subsequent decoding algorithm.

\section{The Traditional Workaround Method Used in DCR System}

To solve the above problem, people put forward some improvement measures $[3,4]$. One method is to try to improve the impedance matching of the circulator port, using Voltage Standing Wave Ratio (VSWR) better antenna switch to increase compensation link in the actual circuit. However, practice shows that the effects of the method are rather limited because of the circulator own property. The second 


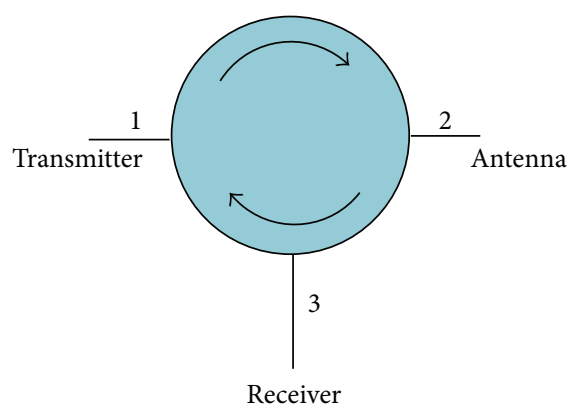

Figure 1: Circulator.

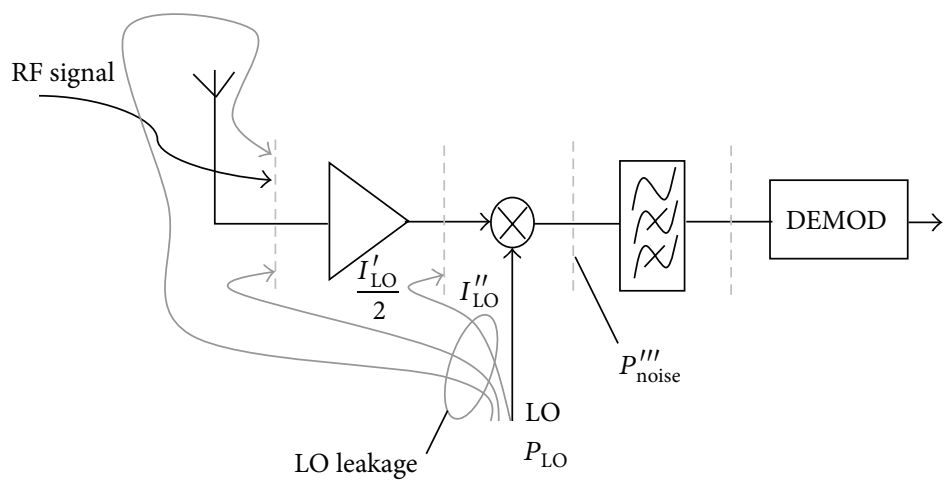

FIGURE 2: Paths of carrier leakage.

method is to reduce the power of the transmitted signal leaks to the receive terminal in order to reduce the transmit signal power level; the result is a reduction in communication distance. The third method, high IP3 double balanced mixers, is used at the receiver; the result is reducing the receiver SNR and also affecting the communication distance and the communication quality at the receiver.

The abovementioned methods are not significantly improving the mentioned problems, and they limit the DCR system scope of the application system.

\section{A Novel Carrier Cancellation Technique}

Carrier cancellation technology is a reverse power synthesis technology [5] that can be used to solve the nonlinear distortion of the power amplifier and extended the measurement frequency limit in super heterodyne spectrum analyzer. Some articles also proposed design methods of carrier cancellation. However, such design methods commonly use lumped parameter approach, and the cancellation signal is generated by the RLC filter, which is generally used in occasion of less than $900 \mathrm{M}$ band. When applied to the higher RF band, the lumped parameter circuit will be seriously affected by distribution parameters, and therefore carrier cancellation function cannot be well implemented.

In order to eliminate the influence of the lumped parameters, a new carrier cancellation method is proposed as shown in Figure 3.

The improved circuit increases the directional coupler, microstrip $180^{\circ}$ phase shifter, and Electric Regulating Control
(ERC) attenuator; the ERC $180^{\circ}$ phase shifter is a key circuit. The following describes the role of each circuit.

(1) Directional Couplers A and B. Two circuits are identical in structure, but different in functions. The role of directional coupler $A$, used as a power divider, is coupled out from a certain energy of RF signal. The role of directional coupler $B$, used as a power combiner, is superposition of the electrical output of the RF modulated signal attenuator and the leakage signal from the circulator and antenna port. Its coupling degree is about $-6 \mathrm{~dB}$, and the center frequency is $2.45 \mathrm{GHz}$ in the paper.

(2) ERC $180^{\circ}$ Phase Shift. This is a key circuit, which not only achieves near $180^{\circ}$ phase shift but also has a very fine phase adjustment capability. Since the carrier phase offset technique is very sensitive in RF frequency, precise matching degree of phase offset will seriously affect the results. In actual implementation, both the leakage signal phase shift and the phase offset cancellation should be considered. Based on the above considerations, we propose using microstrip electronic bridge to realize the phase shifter. To solve the problem of fine-tuning phase, two PIN diodes through an impedance transformation network are placed in the bridge's two arms as voltage control devices. Phase shifter has center frequency of $2.45 \mathrm{GHz}, 3 \mathrm{~dB}$ bandwidth of about $600 \mathrm{MHz}$, and the insertion loss at $2.45 \mathrm{GHz}$ frequency of $3.5 \mathrm{~dB}$. It is shown in Figure 4.

(3) ERC Attenuator. The common PIN diode can be used at lower frequencies, but for high-frequency applications, the 


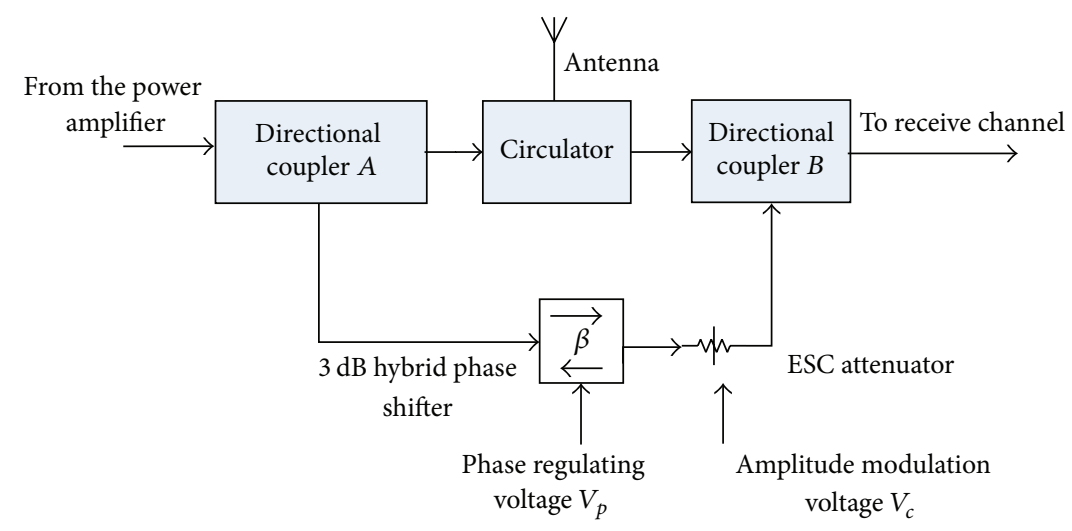

FIgURE 3: Carrier cancellation circuit.

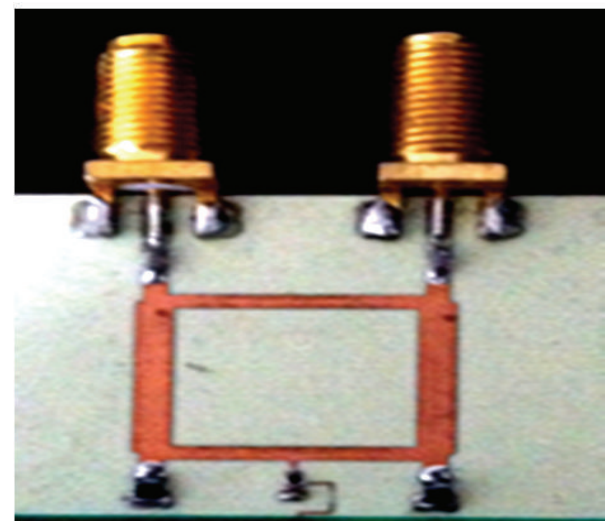

FIGURE 4: ERC $180^{\circ}$ phase shifter.

capacitance of PIN diode junction will reduce the amount of power attenuation. In this paper, the attenuator chip of Skyworks is used to minimize errors. When $V_{c}$ changes $0 \mathrm{~V} \sim 1.2 \mathrm{~V}$, amplitude adjustment range is $-22 \mathrm{~dB} \sim-2 \mathrm{~dB}$. ERC Attenuator cannot only find the best amplitude offset points and is able to adapt to different isolation difference within the circulator.

\section{The ADS Simulation of Carrier Leakage Circuit and Experiment}

Two ports are coupled to each other by the coupling coefficient $M$; the scattering matrix is

$$
\left[\begin{array}{l}
b_{1} \\
b_{2}
\end{array}\right]=\frac{1}{2}\left[\begin{array}{ll}
s_{11} & s_{12} \\
s_{21} & s_{22}
\end{array}\right]\left[\begin{array}{l}
a_{1} \\
a_{2}
\end{array}\right],
$$

where

$$
\begin{aligned}
s_{11}= & T^{2}\left(\alpha_{A}^{2} \Gamma_{A}-\alpha_{B}^{2} \Gamma_{B}\right)-2 j T^{2} M \alpha_{A} \alpha_{B}, \\
s_{12}= & \operatorname{TC}\left(\alpha_{A} \Gamma_{A}-\alpha_{B} \Gamma_{B}\right)+I\left(\alpha_{A}-\alpha_{B}\right) \\
& -j \operatorname{TCM}\left(\alpha_{A}+\alpha_{B}\right),
\end{aligned}
$$

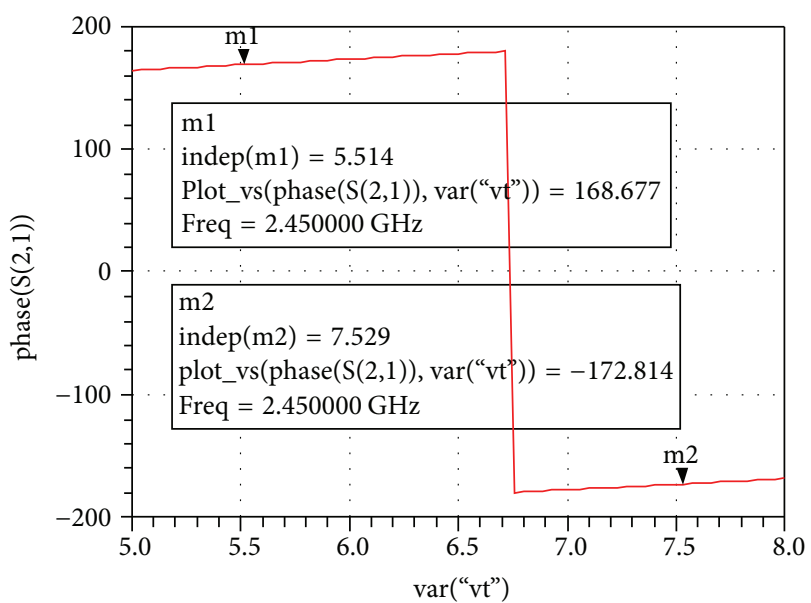

FIGURE 5: The voltage control phase shifter; when the phasecontrolled voltage $V_{p}$ changes at $5.5 \mathrm{~V} \sim 7.5 \mathrm{~V}$, the electrical phase shifter adjusts a phase shift variation of $169.2^{\circ} \sim 187.4^{\circ}$; taking into account the additional phase shift of the circulator and directional coupler itself, the phase control range of the voltage $V_{p}$ may also be larger than the theoretical results, which can meet the overall requirements of the circuit to compensate the phase shift. By carefully adjusting the control voltage $V_{p}$, the exact $180^{\circ}$ phase shift can be obtained between the transmitter port of the circulator and the cancellation signal.

$$
\begin{aligned}
s_{21}= & T C\left(\alpha_{A} \Gamma_{A}-\alpha_{B} \Gamma_{B}\right)+I\left(\alpha_{A}-\alpha_{B}\right) \\
& -j \operatorname{TCM}\left(\alpha_{A}+\alpha_{B}\right), \\
s_{22}= & C^{2}\left(\Gamma_{A}-\Gamma_{B}\right)-2 j C^{2} M .
\end{aligned}
$$

Tx leakage can be expressed as follows:

$$
\begin{aligned}
\frac{b_{2}}{a_{1}} & =\frac{1}{2}\left(T C\left(\alpha_{A} \Gamma_{A}-\alpha_{B} \Gamma_{B}\right)+I\left(\alpha_{A}+\alpha_{B}\right)\right. \\
& \left.-j \operatorname{TCM}\left(\alpha_{A}+\alpha_{B}\right)\right) .
\end{aligned}
$$

The relationship between the voltage control phase shifter is shown in Figure 5. 


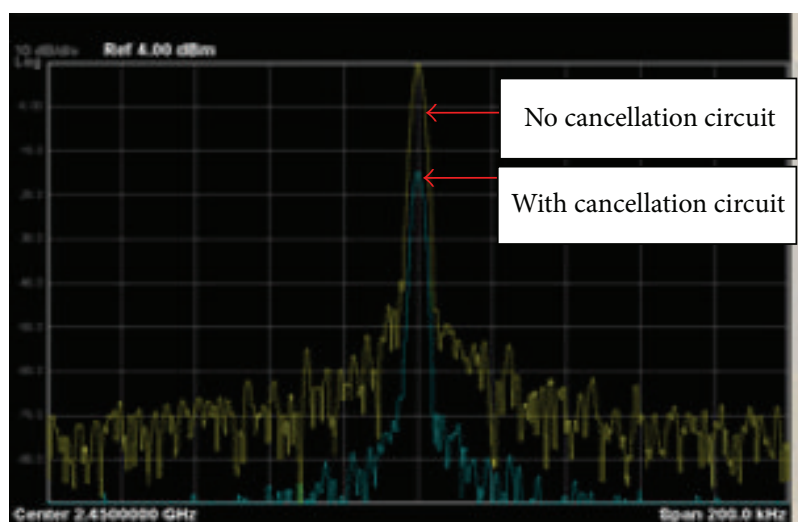

FIgURE 6: Comparison results after adding cancellation circuit.

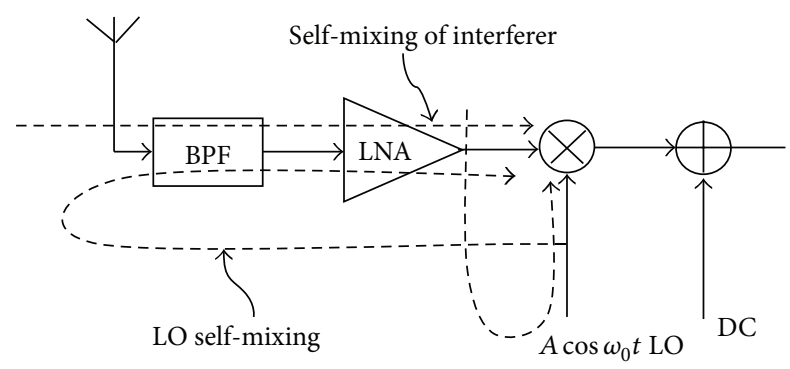

FIGURE 7: Three main sources of leakages give rise to DC offset.

The real result is tested by the spectrum analyzer as shown in Figure 6. Experimental results show that the system can be obtained at $30 \mathrm{db}$ attenuation.

\section{DC Offset Problems and Solutions in DCR System}

DCR receiver will bring unwanted DC component; it is much larger than the useful baseband signal amplitude. Three main sources of leakages give rise to DC offset as shown in Figure 7. For example, if the receiver front-end mixer LO level is $+17 \mathrm{dBm}$, transmit power is $1 \mathrm{~W}$ and circulator isolation is about $20 \mathrm{~dB}$. When not using the carrier cancellation circuit, the leakage to the mixer local oscillator signal power is up to $10 \mathrm{~mW}$.

The DC offset voltage is attained:

$$
\begin{aligned}
V_{m} & =k_{m} \times\left(V_{\mathrm{DCI}}+V_{I}\right) \times\left(V_{\mathrm{DCQ}}+V_{\mathrm{Q}}\right)=k_{m} \times\left[V_{\mathrm{DCI}}\right. \\
& \left.\times V_{\mathrm{DCQ}}+\left(V_{I} \times V_{\mathrm{DCQ}}+V_{\mathrm{Q}} \times V_{\mathrm{DCI}}+V_{I} \times V_{\mathrm{Q}}\right)\right] \\
& =U+W,
\end{aligned}
$$

where $k_{m}$ denotes the amplifying coefficient, and

$$
\begin{aligned}
& U=k_{m} \times V_{\mathrm{DCI}} \times V_{\mathrm{DCQ}}, \\
& W=k_{m} \times\left(V_{I} \times V_{\mathrm{DCQ}}+V_{\mathrm{Q}} \times V_{\mathrm{DCI}}+V_{I} \times V_{\mathrm{Q}}\right),
\end{aligned}
$$

where $U$ is DC signal and $W$ is signal including both DC and AC components.

Taking into account the conversion $6 \mathrm{db}$ loss of the mixer, the DC voltage output from the mixer is about $447 \mathrm{mV}$. With the carrier cancellation circuit, the DC voltage output of the mixer is about $30 \mathrm{mV}$, and the DC voltage is larger than the amplitude of the baseband signal.

Due to the presence of the DC voltage, an AC-coupled amplifier is generally used to amplify the weak baseband signal. Theoretical analysis shows that AC coupling will have an impact on the quality of the digital baseband signal. Figure 8 shows the result of the different data rate using multistage AC amplifier.

For a fixed coupling constant AC amplifier, the special data rate is appropriate for the special data rate, but when the rate of the baseband signal is higher or lower than the frequency, amplified signal will produce integral or differential effects, and the baseband signal integrity is damaged, so some complex software algorithms were designed to correct the distorted baseband signals [6-9].

\section{A New Wideband DC Amplifier Design with DC Feedback Loop}

To solve these problems, we proposed a new novel wideband and high gain amplifier with low frequency servo loop. The basic circuit is shown in Figure 9.

The circuits contain two low-pass filters, a multistage wideband DC amplifier, an integrator, and an active threshold decision circuit. The high-frequency signal of mixer is filtered by the low-pass filter 1 , and baseband and DC signal are retained. The multistage DC amplifiers have large dynamic scope; the gain is set at $100 \mathrm{db}$ in the simulation circuit, and the full-power bandwidth of amplifier satisfies the maximum rate requirements of DCR system. The low-pass filter 2 and the integrator circuit are the core components to cancel the effect of DC and $1 / f$ noise; the circuits filter out the AC component from the DC amplifier while retaining its DC and low frequency components; the active integrator accumulates the DC voltage and feed back to the inverse input of the high-speed DC amplifier, because the integrator samples the signal from the last amplifier. The DC component produced by the front mixer or by the high-speed operational amplifier self will be limited to a very small level. Taking into account $1 / f$ noise in wideband amplifier, the low-pass filter 2 and integrator must be set at a higher cut-off frequency, but in order to try to maintain the integrity of the baseband signal, the cut-off frequency should be set lower.

The baseband signal is series rectangle pulse, and according to Fourier transform, the rectangular pulse can be expressed as

$$
\begin{aligned}
\left|F_{n}\right| & =\frac{1}{2} \sqrt{a_{n}^{2}+b_{n}^{2}}=\frac{1}{2} \sqrt{\frac{4 \sin ^{2}\left(n \omega_{0} \tau / 2\right)}{n^{2} \pi^{2}}} \\
& =\frac{1}{2} \cdot \frac{2 \sin \left(n \omega_{0} \tau / 2\right)}{n \pi}=\frac{\sin \left(n \omega_{0} \tau / 2\right)}{n \pi} .
\end{aligned}
$$




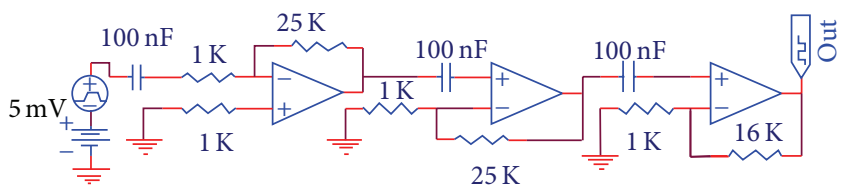

(a) High gain AC amplifier circuit

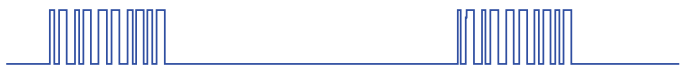

(b) Input coded signal

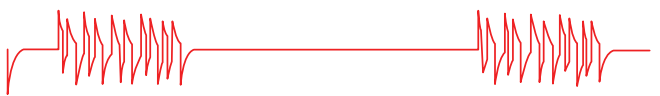

(c) The distortion signal after AC amplifier in low data rate

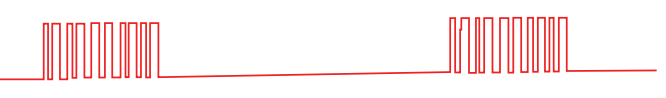

(d) Amplified signal exactly matches AC coupling parameter

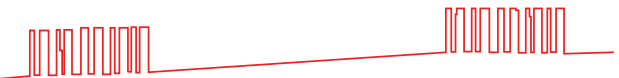

(e) The distortion signal after AC amplifier in high data rate

FIgURE 8: AC amplification distortions.

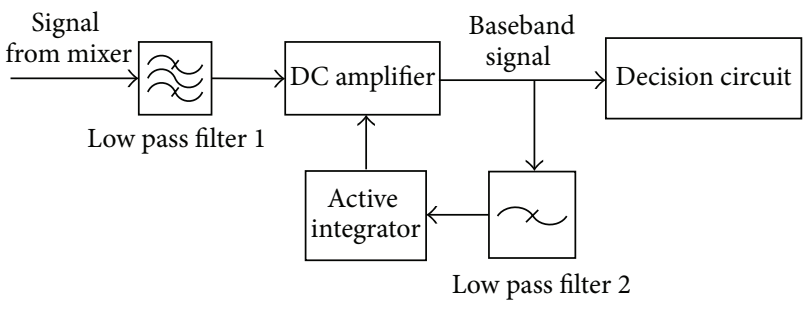

Figure 9: DC amplifier circuit with low frequency servo loop.

Power spectrum is expressed as

$$
\begin{aligned}
\varphi(\omega) & =2 \pi \cdot \sum_{n=-\infty}^{\infty} \frac{\sin ^{2}\left(n \omega_{0} \tau / 2\right)}{n^{2} \pi^{2}} \delta\left(\omega-n \omega_{0}\right) \\
& =2 \pi \sum_{n=-\infty}^{\infty} \frac{\sin ^{2}(n \pi \cdot(\tau / T))}{n^{2} \pi^{2}} \delta\left(\omega-n \omega_{0}\right) .
\end{aligned}
$$

The average normalized power spectral density of a series of rectangular pulses representing $n$ data bits is thus

$$
\begin{aligned}
G(f) & =\frac{n A^{2} \tau^{2} \sin c^{2}(\pi f \tau)}{n \tau} \\
& =A^{2} \tau \sin c^{2}(\pi f \tau) \text { volts } 2 / \mathrm{Hz}
\end{aligned}
$$

Generally, the percentage of signal's power within a frequency band is a good indication of the signal distortion. By the circuit simulation result, it is appropriate that the cut-off frequency is set at $0.1 \%$ of the baseband data rate; for example, if the data rate is $32 \mathrm{kHz}$, the cut-off frequency of low-pass filter 2 can be set at $32 \mathrm{~Hz}$.

At the same time, the cut-off frequency determines the offset $1 / f$ noise; according to the typical MOSFET amplifiers model, the $1 / f$ noise in the vicinity of $1 \mathrm{KHz}$ can be expressed as

$$
\left.\frac{K}{W L C_{\mathrm{OX}}} \cdot \frac{1}{f}\right|_{f \approx 1 \mathrm{KHz}} \approx 4 k T \frac{2}{3 g_{m}} .
$$

The $1 / f$ noise power in a bandwidth from $0 \mathrm{~Hz}$ to $32 \mathrm{~Hz}$ can be calculated:

$$
\begin{aligned}
P_{n 1} & =\int_{0 \mathrm{~Hz}}^{32 \mathrm{~Hz}} \frac{K}{W L C_{\mathrm{OX}}} \cdot \frac{1}{f} d f=\frac{K}{W L C_{\mathrm{OX}}} \ln 32 \\
& =4 k T \frac{2}{3 g_{m}}(1 \mathrm{KHz}) \ln 32 .
\end{aligned}
$$

For the thermal noise, we have

$$
\begin{aligned}
P_{n 2} & =\int_{0 \mathrm{~Hz}}^{32 \mathrm{~Hz}} \frac{K}{W L C_{\mathrm{OX}}} d f=\int_{0 \mathrm{~Hz}}^{32 \mathrm{~Hz}} 4 k T \frac{2}{3 g_{m}} d f \\
& =4 k T \frac{2}{3 g_{m}} \cdot 32 .
\end{aligned}
$$

Thus, the cancellation noise power is

$$
\frac{P_{n 1}}{P_{n 2}}=108.1=20.3 \mathrm{~dB} .
$$

Detailed circuits are shown in Figure 10; compared to the AC coupling model (Figure 8), the improved circuit is DC coupling mode with low frequency feedback servo loop. The multistage amplifier gain is set at $100 \mathrm{db}$ in the simulation, and the Manchester coded data with $5 \mathrm{mV}$ DC offset are applied to both circuits.

The simulation results (Figure 11) show that both circuits can remove the DC offset, but the AC coupling amplifier cannot adapt various data rate and causes signal distortion at high data rate and low data rate; while DC coupling amplifier with low frequency servo loop can keep the signal integrity at any data speed, the signal has no significant distortion.

This circuit is applied in DCR RFID system, and the test result is shown in Figure 12; the wave of baseband signal reflected from the tag can be very well maintained in different data rate.

\section{Conclusion}

This paper presents the design of microstrip circuit carrier cancellation technology for DCR system; experimental 


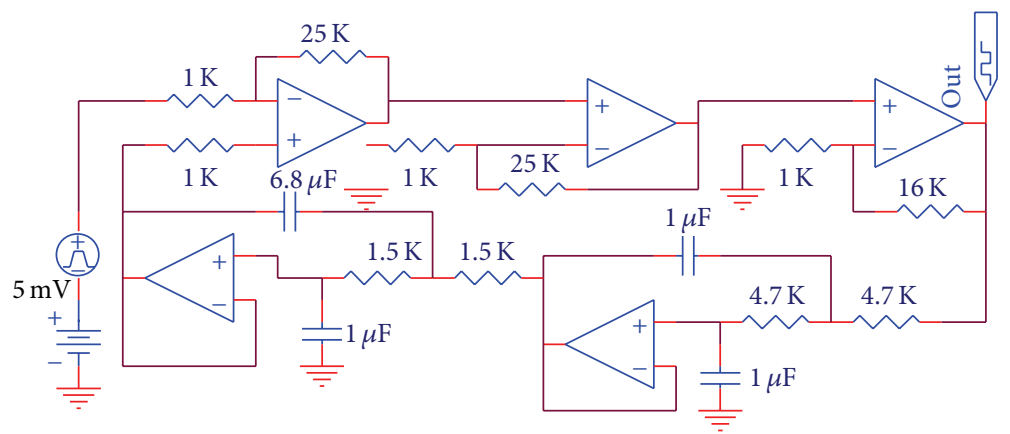

FIGURE 10: Improved DC coupling circuit with low frequency servo loop based on discrete components.

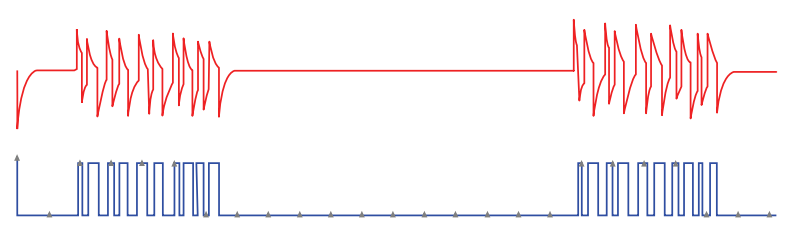

(a) $16 \mathrm{kbps}$ data rate

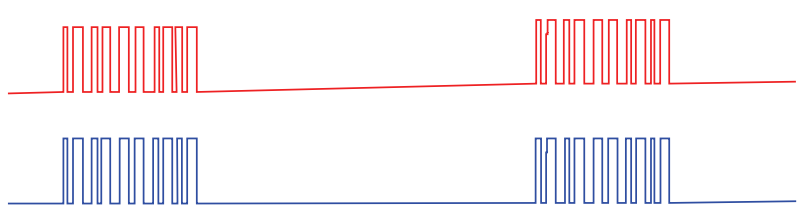

(b) 160 kbps data rate

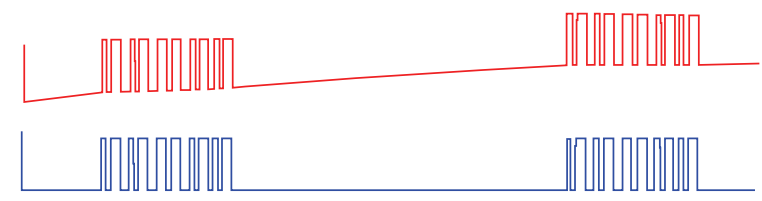

(c) $320 \mathrm{kbps}$ data rate

Figure 11: (a), (b), and (c) show the comparison results of AC coupling (red color) and improved DC coupling amplifier (blue color) in $16 \mathrm{kbps}, 160 \mathrm{kbps}$, and $320 \mathrm{kbps}$ data rate.

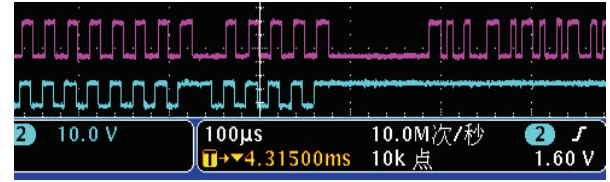

(a)

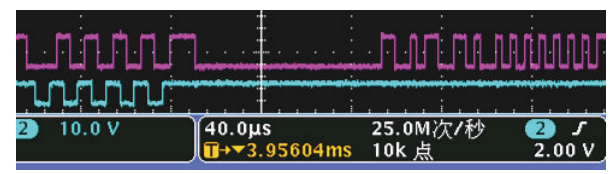

(b)

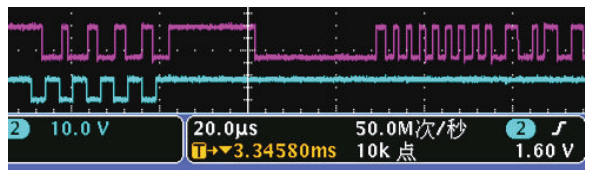

(c)

FIGURE 12: Measured waveform of the reader and tag: pink color (top) is tag reflected signal, and blue color (bottom) is reader command. (a), (b), and (c) are $40 \mathrm{kbps}, 80 \mathrm{kbps}$, and $160 \mathrm{kbps}$ data rate.

results show that the use of this technology solution can reduce the carrier leakage power $25 \mathrm{~dB}$, equivalent to the circulator isolation up to $45 \mathrm{~dB}$. This approach ensures the linearity of the receiver, so that more low noise preamplifiers can be used in the front-end circuit of the receiver to increase the communication distance of DCR system. Further, for the problem of DC offset, a new wideband amplifier circuit with DC stereo loop is proposed; the simulation and experimental results show that the novel circuits can solve the DC offset problem and keep the integrity of baseband signal.

\section{Competing Interests}

The authors declare that they have no competing interests.

\section{Acknowledgments}

This work was supported by the National Research Foundation of China grant funded by the China government (11374162) and University Natural Science Project (TJ215009, NY215162). Thanks are due for the help of Senior Engineer Jingqing Cheng and Yufeng Guo.

\section{References}

[1] B. Razavi, "Design considerations for direct-conversion receivers," IEEE Transactions on Circuits and Systems II: Analog and Digital Signal Processing, vol. 44, no. 6, pp. 428-435, 1997.

[2] W.-G. Lim and J.-W. Yu, "Balanced circulator structure with enhanced isolation characteristics," Microwave and Optical Technology Letters, vol. 50, no. 9, pp. 2389-2391, 2008. 
[3] A. Yoshizawa and Y. P. Tsividis, "Anti-blocker design techniques for MOSFET-C filters for direct conversion receivers," IEEE Journal of Solid-State Circuits, vol. 37, no. 3, pp. 357-364, 2002.

[4] K.-J. Cho, J.-H. Kim, and S. P. Stapleton, "A highly efficient doherty feedforward linear power amplifier for W-CDMA basestation applications," IEEE Transactions on Microwave Theory and Techniques, vol. 53, no. 1, pp. 292-300, 2005.

[5] M. Grimm, M. Allén, J. Marttila, M. Valkama, and R. Thomä, "Joint mitigation of nonlinear RF and baseband distortions in wideband direct-conversion receivers," IEEE Transactions on Microwave Theory and Techniques, vol. 62, no. 1, pp. 166-182, 2014.

[6] G. Byeon, S. Oh, and G. Jang, "A new DC offset removal algorithm using an iterative method for real-time simulation," IEEE Transactions on Power Delivery, vol. 26, no. 4, pp. 22772286, 2011.

[7] L. Yu and W. M. Snelgrove, "A novel adaptive mismatch cancellation system for quadrature if radio receivers," IEEE Transactions on Circuits and Systems II: Analog and Digital Signal Processing, vol. 46, no. 6, pp. 789-801, 1999.

[8] H. Yoshida, H. Tsurumi, and Y. Suzuki, "DC offset canceller in a direct conversion receiver for QPSK signal reception," in Proceedings of the 9th IEEE International Symposium on Personal, Indoor and Mobile Radio Communications, vol. 3, pp. 1314-1318, IEEE, Boston, Mass, USA, September 1998.

[9] J.-Y. Jung, C.-W. Park, and K.-W. Yeom, "A novel carrier leakage suppression front-end for UHF RFID reader," IEEE Transactions on Microwave Theory and Techniques, vol. 60, no. 5, pp. 1468-1477, 2012. 


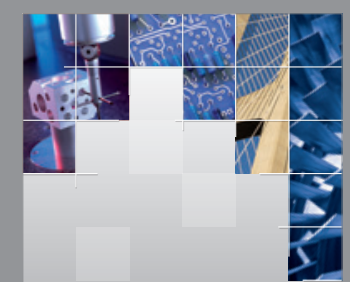

\section{Enfincering}
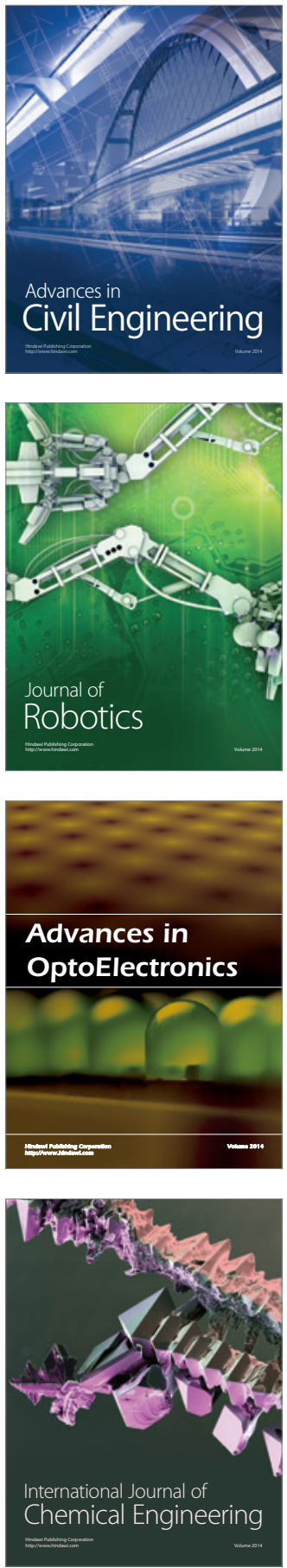

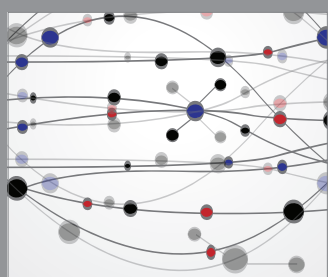

The Scientific World Journal

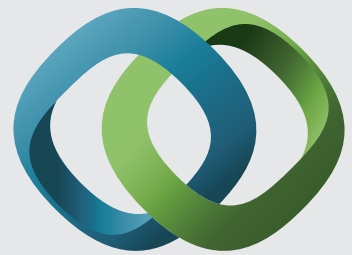

\section{Hindawi}

Submit your manuscripts at

http://www.hindawi.com
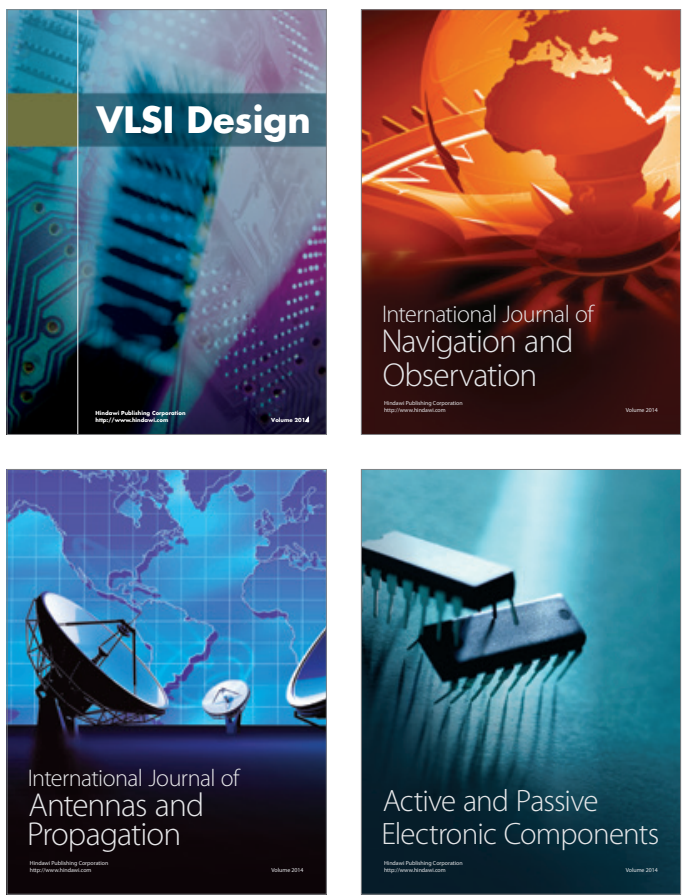
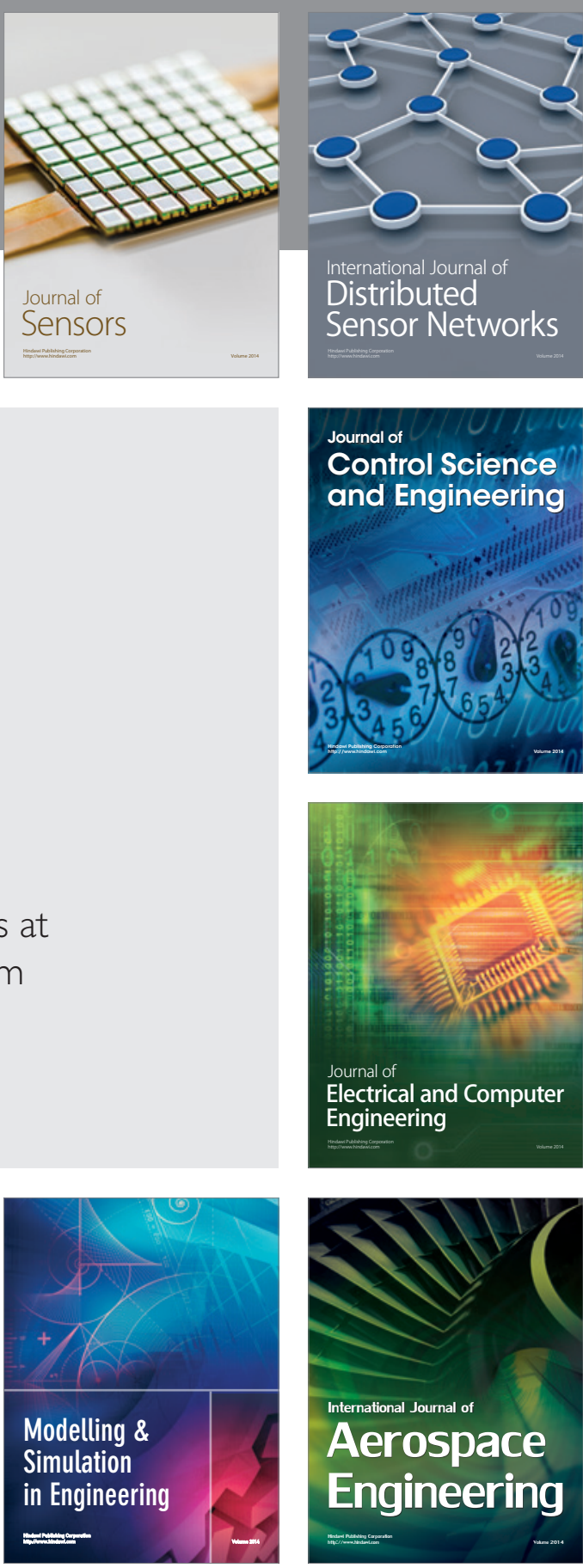

International Journal of

Distributed

Sensor Networks

Journal of

Control Science

and Engineering
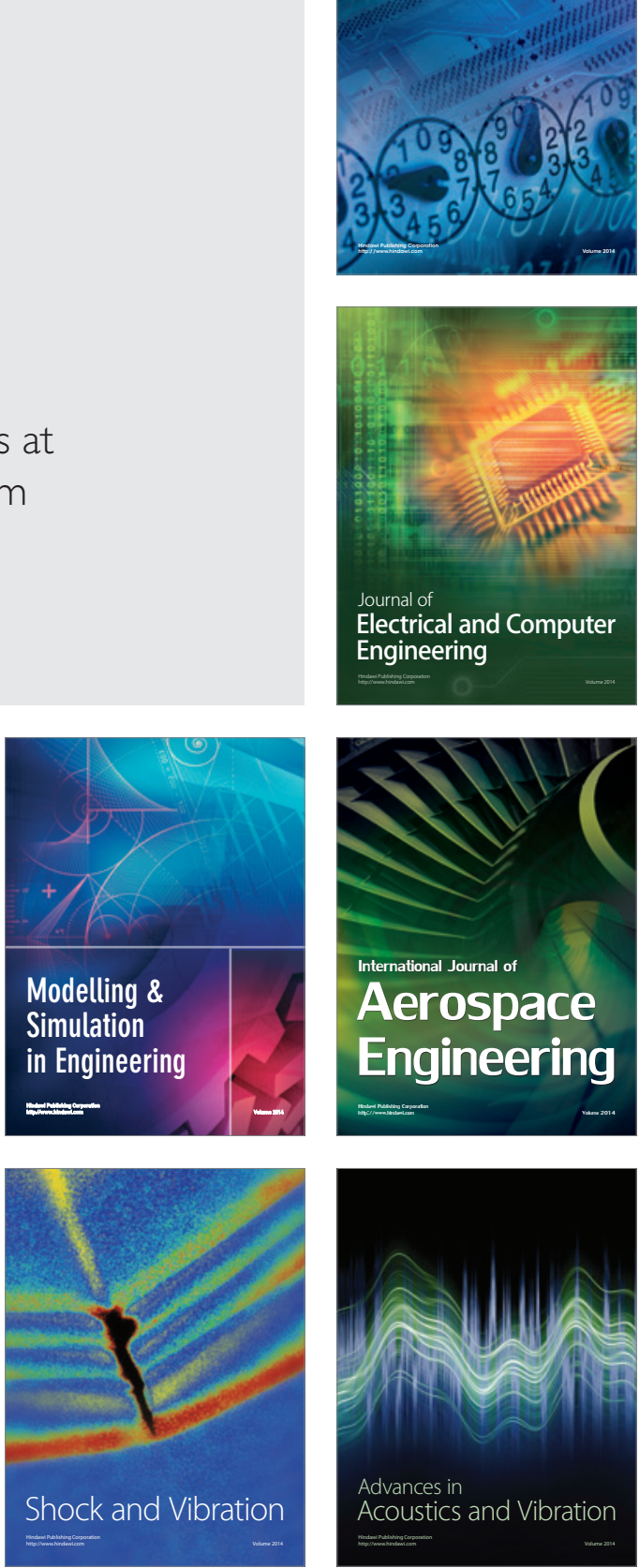